\title{
Superfluid Helium in Three-Dimensional Counterflow Differs Strongly from Classical Flows : Anisotropy on Small Scales
}

\section{Biferale, L.}

2019-04-08

Biferale , L , Khomenko , D , L'vov , V , Pomyalov , A \& Sahoo , G 2019 , ' Superfluid Helium in Three-Dimensional Counterflow Differs Strongly from Classical Flows : Anisotropy on

Small Scales ' , Physical Review Letters , vol. 122 , no. 14 , 144501 . https://doi.org/10.1103/PhysRevLett.122.14450

http://hdl.handle.net/10138/300960

https://doi.org/10.1103/PhysRevLett.122.144501

cc_by

publishedVersion

Downloaded from Helda, University of Helsinki institutional repository.

This is an electronic reprint of the original article.

This reprint may differ from the original in pagination and typographic detail.

Please cite the original version. 


\title{
Superfluid Helium in Three-Dimensional Counterflow Differs Strongly from Classical Flows: Anisotropy on Small Scales
}

\author{
L. Biferale, ${ }^{1}$ D. Khomenko, ${ }^{2}$ V. L'vov, ${ }^{3}$ A. Pomyalov, ${ }^{3}$ I. Procaccia,${ }^{3}$ and G. Sahoo ${ }^{4}$ \\ ${ }^{1}$ Department of Physics and INFN, University of Rome, Tor Vergata, 00133 Roma, Italy \\ ${ }^{2}$ Laboratoire de physique l'Ecole normale supérieure, CNRS, 75005 Paris, France \\ ${ }^{3}$ Department of Chemical and Biological Physics, Weizmann Institute of Science, 7610001 Rehovot, Israel \\ ${ }^{4}$ Department of Mathematics and Statistics and Department of Physics, University of Helsinki, FI-00014 Helsinki, Finland
}

(Received 9 January 2019; revised manuscript received 26 February 2019; published 8 April 2019)

\begin{abstract}
Three-dimensional anisotropic turbulence in classical fluids tends towards isotropy and homogeneity with decreasing scales, allowing-eventually-the abstract model of homogeneous and isotropic turbulence to be relevant. We show here that the opposite is true for superfluid ${ }^{4} \mathrm{He}$ turbulence in three-dimensional counterflow channel geometry. This flow becomes less isotropic upon decreasing scales, becoming eventually quasi-two-dimensional. The physical reason for this unusual phenomenon is elucidated and supported by theory and simulations.
\end{abstract}

DOI: $10.1103 /$ PhysRevLett.122.144501

All turbulent flows in nature and in laboratory are anisotropic on the energy injection scales [1]. Nevertheless, the model of homogeneous and isotropic turbulence (HIT) has been shown to be highly successful in predicting the statistical properties of turbulence on scales much smaller than stirring scales (but still larger than the dissipative scale). The reason for this lies in the nature of the nonlinear terms of the equations of fluid mechanics that tend to isotropize the flow upon cascading energy to smaller scales. Eventually, at small enough scales, the flow becomes sufficiently isotropic to allow the application of HIT [2]. In the present Letter, we show that in turbulent superfluid ${ }^{4} \mathrm{He}$ in a channel with a temperature gradient, the opposite phenomenon takes place: the flow becomes less and less isotropic upon decreasing the scales. Eventually, the flow becomes quasi-two-dimensional with interesting and unusual properties as detailed below. A possible way to account for this difference is furnished by the two-fluid model of superfluid ${ }^{4} \mathrm{He}$ turbulence [3-5]. We denote by $\boldsymbol{u}_{\mathrm{s}}$ and $\boldsymbol{u}_{\mathrm{n}}$ the superfluid and normal-fluid velocities, respectively. In counterflow geometry, with a temperature gradient directed along the channel, the superfluid flows towards the heater and the normal fluid away from the heater. Importantly, there exists a mutual friction force $f_{\mathrm{ns}}$ between these two components [4-9], proportional to the difference in velocities, i.e., $\boldsymbol{f}_{\mathrm{ns}} \propto\left(\boldsymbol{u}_{\mathrm{n}}-\boldsymbol{u}_{\mathrm{s}}\right)$. As long as the fluctuations between these two velocities are correlated, this force remains small. Upon the loss of correlation, this force becomes large and will lead to a suppression of the corresponding fluctuations. Consider then two types of velocity fluctuations, one elongated along the channel and the counterflow, and the other orthogonal to them; see Fig. 1. Due to the mean flow in opposite directions, the velocity fluctuations oriented orthogonally will have a short overlap time and will decorrelate quickly, whereas the velocity fluctuations along the counterflow will remain correlated for a longer time. The result will be a strong suppression of the former with respect to the latter. This will eventually lead to a turbulent flow in which the fluctuations consist mostly of the streamwise component, while the energy is concentrated in the plane orthogonal to the counterflow direction. The rest of this Letter will elaborate this picture by using an analytical approach and using direct numerical simulations (DNS).

The basic equations. - The two-fluid model describes superfluid ${ }^{4} \mathrm{He}$ of density $\rho=\rho_{\mathrm{s}}+\rho_{\mathrm{n}}$ as a mixture of two interpenetrating fluid components: an inviscid superfluid, $\rho_{\mathrm{s}}$, and a viscous normal fluid, $\rho_{\mathrm{n}}$. The fluid components are coupled by a mutual friction force, mediated by the tangle of

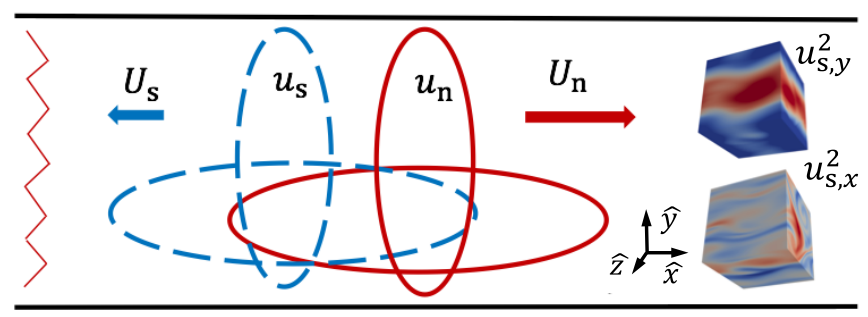

FIG. 1. Schematics of the superfluid ${ }^{4} \mathrm{He}$ channel counterflow. The normal-fluid (solid red lines) and the superfluid eddies (blue dashed lines) are swept by the corresponding mean velocities $U_{\mathrm{n}}$ and $U_{\mathrm{s}}$ away and towards the heater, respectively. The streamwiseelongated eddies have longer overlap time than the cross-streamelongated eddies. Right inset: Volume rendering of the squared superfluid velocity components $u_{\mathrm{s}, y}^{2}$ (top) and $u_{\mathrm{s}, x}^{2}$ (bottom). The $u_{\mathrm{s}, z}$ (not shown) is similar to the $y$ component. The velocity magnitude is color coded, with red denoting positive and blue denoting negative values. 
quantum vortices [4-8] of a core radius $a_{0} \approx 10^{-8} \mathrm{~cm}$ and a fixed circulation $\kappa=h / M \approx 10^{-3} \mathrm{~cm}^{2} / \mathrm{s}$, where $h$ is Planck's constant and $M$ is the mass of the ${ }^{4} \mathrm{He}$ atom [10]. A complex tangle of these vortex lines with a typical intervortex distance [5] $\ell \sim 10^{-4}-10^{-2} \mathrm{~cm}$ is a manifestation of superfluid turbulence.

We employ the coarse-grained equations for counterflow [11-15]. They have a form of two Navier-Stokes equations for the velocity fluctuations $\boldsymbol{u}_{j}(\boldsymbol{r}, t)$ of the normal fluid $(j=\mathrm{n})$ and the superfluid $(j=\mathrm{s})$ :

$$
\left(\frac{\partial}{\partial t}+\left(\boldsymbol{u}_{j}+\boldsymbol{U}_{j}\right) \cdot \boldsymbol{\nabla}\right) \boldsymbol{u}_{j}-\frac{\boldsymbol{\nabla} p_{j}}{\rho_{j}}=\nu_{j} \Delta \boldsymbol{u}_{j}+\boldsymbol{f}_{j}+\boldsymbol{\varphi}_{j},
$$

coupled by the friction $f_{j}$ in the minimal form [16]:

$\boldsymbol{f}_{\mathrm{s}} \simeq \Omega_{\mathrm{s}}\left(\boldsymbol{u}_{\mathrm{n}}-\boldsymbol{u}_{\mathrm{s}}\right), \quad \boldsymbol{f}_{\mathrm{n}} \simeq \Omega_{\mathrm{n}}\left(\boldsymbol{u}_{\mathrm{s}}-\boldsymbol{u}_{\mathrm{n}}\right), \quad \Omega_{\mathrm{s}}=\alpha \kappa \mathcal{L}$

and $\Omega_{\mathrm{n}}=\rho_{\mathrm{s}} \Omega_{\mathrm{s}} / \rho_{\mathrm{n}}$. The mutual friction frequency $\Omega_{\mathrm{s}}$ depends on the temperature-dependent dimensionless mutual friction parameter $\alpha(T)$ and on the vortex line density (VLD) $\mathcal{L}$. In Eq. (1), $\boldsymbol{U}_{j}$ are the mean velocities and $p_{j}$ are the pressures of the normal-fluid and the superfluid components. The kinematic viscosity of the normal fluid is $\nu_{\mathrm{n}}=\eta / \rho_{\mathrm{n}}$, with $\eta$ being the dynamical viscosity of ${ }^{4} \mathrm{He}$ [17]. The energy sink in the equation for the superfluid component, proportional to the effective superfluid viscosity, $\nu_{\mathrm{s}}$, accounts for the energy dissipation at the intervortex scale $\ell$, due to vortex reconnections and energy transfer to Kelvin waves $[5,11,18]$.

The large-scale motion in the thermal counterflow is sustained by the temperature gradient, created along the channel. Here we use the fact that the center of the channel flow at large enough Reynolds numbers can be considered as almost space-homogeneous [19]. To simplify the analysis, we consider homogeneous turbulence under periodic boundary conditions and mimic the steering of turbulence at large scales by random forces $\boldsymbol{\varphi}_{j}$. Equations (1a) and (1b) describe the motion of two fluid components in the range of scales between the forcing scale and the intervortex distance. Ideally, to accurately describe dynamics of the system, a set of closed coupled equations for $\boldsymbol{u}_{\mathrm{s}}, \boldsymbol{u}_{\mathrm{n}}$, and $\mathcal{L}$ should be solved. However, currently there is no consensus on what equations must be used for the VLD and whether $\mathcal{L}$ is the only tangle property relevant for the vortex dynamics [20-23]. Here we use $\Omega_{\mathrm{s}}$ in Eq. (1b) as a control parameter, which is a reasonable approximation for homogeneous turbulence under periodic boundary conditions. Note that numerical simulations of Eqs. (1a) and (1b) were used to successfully predict the enhancement of small-scale intermittency in the mechanically driven superfluid ${ }^{4} \mathrm{He}$ [11], recently confirmed experimentally in Ref. [24]. Similarly, the energy spectra of counterflow turbulence, analytically derived [15] using Eq. (1), are in a good agreement with the experiment of Refs. [25,26].
Statistics of anisotropic turbulence.-The most general description of homogeneous superfluid ${ }^{4} \mathrm{He}$ turbulence at the level of second-order statistics can be done in terms of the three-dimensional (3D) Fourier spectrum of each component and the cross-correlation functions:

$$
(2 \pi)^{3} \delta\left(\boldsymbol{k}-\boldsymbol{k}^{\prime}\right) \mathcal{F}_{i j}^{\alpha \beta}(\boldsymbol{k})=\left\langle v_{i}^{\alpha}(\boldsymbol{k}) v_{j}^{* \beta}\left(\boldsymbol{k}^{\prime}\right)\right\rangle,
$$

where $\boldsymbol{\nu}_{j}(\boldsymbol{k})$ is the Fourier transform of $\boldsymbol{u}_{j}(\boldsymbol{r})$, the indices $i$ and $j$ refer to the fluid components, the vector indices $\alpha, \beta=$ $\{x, y, z\}$ denote the Cartesian coordinates, and ${ }^{*}$ stands for complex conjugation. In the following, we choose the counterflow velocity, $U_{\mathrm{ns}}=U_{\mathrm{n}}-U_{\mathrm{s}}$ along the $\hat{\boldsymbol{x}}$ direction as depicted in Fig. 1. Next, we denote the trace of any tensor according to $\mathcal{F}_{j j}(\boldsymbol{k}) \equiv \sum_{\alpha} \mathcal{F}_{j j}^{\alpha \alpha}(\boldsymbol{k})$. With this notation, the kinetic energy density per unit mass $\mathcal{E}_{j}$ reads

$$
\mathcal{E}_{j} \equiv \frac{1}{2}\left\langle\left|\boldsymbol{u}_{j}(\boldsymbol{r})\right|^{2}\right\rangle=\frac{1}{2} \int \mathcal{F}_{j j}(\boldsymbol{k}) d^{3} k /(2 \pi)^{3} .
$$

Due to the presence of the preferred direction, defined by $\boldsymbol{U}_{\text {ns }}$, the counterflow turbulence has an axial symmetry around the $\hat{\boldsymbol{x}}$ axis. Then $\mathcal{F}_{i j}(\boldsymbol{k})$ depends only on the two projections $k_{\|}=k_{x}$ and $k_{\perp}=\sqrt{k_{y}^{2}+k_{z}^{2}}$ of the wave vector $\boldsymbol{k}$, being independent of the angle $\phi$ in the plane, orthogonal to $\boldsymbol{U}_{\mathrm{ns}}$. This allows us to define a set of two-dimensional (2D) objects that still contain all the information about second-order statistics of the counterflow turbulence:

$$
F_{i j}\left(k_{\|}, k_{\perp}\right) \equiv \frac{k_{\perp}}{4 \pi^{2}} \mathcal{F}_{i j}\left(k_{\|}, k_{\perp}\right) .
$$

Another way to represent the same information is to introduce a polar angle, $\cos (\theta)=\left(\boldsymbol{k}, \boldsymbol{U}_{\mathrm{ns}}\right) /|\boldsymbol{k}|\left|\boldsymbol{U}_{\mathrm{ns}}\right|$, and to use spherical coordinates:

$$
\tilde{F}_{i j}(k, \theta) \equiv \frac{k}{4 \pi^{2}} \mathcal{F}_{i j}(k \cos \theta, k \sin \theta) .
$$

Physical origin of the strong anisotropy.-The physical origin of the strong anisotropy in the counterflow turbulence is best exposed by considering the balance equation for the 2D energy spectra $\tilde{F}_{\text {nn }}(k, \theta), \tilde{F}_{\text {ss }}(k, \theta)$. For that, we start with Eq. (1), follow the procedure described in Ref. [15], and average the resulting equations for the 3D spectra over the azimuthal angle $\varphi$. Finally, for the normal-fluid component, we get

$$
\begin{aligned}
\frac{\partial \tilde{F}_{\mathrm{nn}}(k, \theta, t)}{\partial t}+\operatorname{div}_{\boldsymbol{k}}\left[\boldsymbol{\varepsilon}_{\mathrm{n}}(\boldsymbol{k})\right] & =-\mathcal{D}_{\mathrm{n}}^{\mathrm{mf}}(k, \theta)-\mathcal{D}_{\mathrm{n}}^{\mathrm{kv}}(k, \theta), \\
\mathcal{D}_{\mathrm{n}}^{\mathrm{mf}}(k, \theta) & =\Omega_{\mathrm{n}}\left[\tilde{F}_{\mathrm{nn}}(k, \theta)-\tilde{F}_{\mathrm{ns}}(k, \theta)\right], \\
\mathcal{D}_{\mathrm{n}}^{\mathrm{kv}}(k, \theta) & =2 \nu_{\mathrm{n}} k^{2} \tilde{F}_{\mathrm{nn}}(k, \theta),
\end{aligned}
$$

where $\operatorname{div}_{\boldsymbol{k}}\left[\boldsymbol{\varepsilon}_{j}(\boldsymbol{k})\right]$ is the transfer term due to inertial nonlinear effects, and $\mathcal{D}_{\mathrm{n}}^{\mathrm{mf}}(k, \theta)$ describes the rate of energy 
dissipation by the mutual friction, while $\mathcal{D}_{\mathrm{n}}^{\mathrm{kv}}(k, \theta)$ stands for the rate of dissipation by the kinematic viscosity. A similar equation is obtained for the superfluid component by replacing "n" with "s" everywhere. For a qualitative analysis of the origin of the anisotropy in our system, it is important to develop a closure of the cross-correlation function $\tilde{F}_{\text {ns }}(k, \theta)$ in $\mathcal{D}_{j}^{\mathrm{mf}}(k, \theta)$ in terms of the spectral properties of each fluid component and of the counterflow velocity.

According to Ref. [12],

$$
\tilde{F}_{\mathrm{ns}}(k, \theta)=A B /\left[B^{2}+\left(\boldsymbol{k} \cdot \boldsymbol{U}_{\mathrm{ns}}\right)^{2}\right] .
$$

Here $A=\Omega_{\mathrm{s}} \tilde{F}_{\mathrm{nn}}(k, \theta)+\Omega_{\mathrm{n}} \tilde{F}_{\mathrm{ss}}(k, \theta)$ and $B$ can be approximated as $B=\Omega_{\mathrm{n}}+\Omega_{\mathrm{s}}$, as shown in Ref. [15]. We further simplify $\tilde{F}_{\text {ns }}(k, \theta)$ in Eq. (6) by noting [15] that when two components are highly correlated, the cross-correlation may be accurately represented by the corresponding energy spectra. For wave numbers where the components are not correlated, as is quantified by the decorrelation function $D(k, \theta)$ [12], $\tilde{F}_{\text {ns }}(k, \theta)$ is small, and the accuracy of its representation is less important. We therefore get a decoupled form of the cross-correlation:

$$
\begin{gathered}
\tilde{F}_{\mathrm{ns}}(k, \theta)=\tilde{F}_{j j}(k, \theta) D(k, \theta), \\
D(k, \theta)=\left[1+\left(\frac{k U_{\mathrm{ns}} \cos \theta}{\Omega_{\mathrm{n}}+\Omega_{\mathrm{s}}}\right)^{2}\right]^{-1},
\end{gathered}
$$

and finally determine the rate of energy dissipation due to mutual friction:

$$
\mathcal{D}_{j}^{\mathrm{mf}}(k, \theta)=\Omega_{j} \tilde{F}_{j j}(k, \theta)[1-D(k, \theta)] .
$$

Equations (7a)-(7c) are the central analytical result of this paper.

The impact of $U_{\mathrm{ns}}$ on the anisotropy follows from the closure (7c). Indeed, for small $k$ or even for large $k$ with $\boldsymbol{k}$ almost perpendicular to $\boldsymbol{U}_{\text {ns }}$ (i.e., $\left.\cos \theta \ll 1\right), D(k, \theta) \simeq 1$, the normal-fluid and superfluid velocities are almost fully coupled, and the dissipation rate is small: $\mathcal{D}_{j}^{\mathrm{mf}}(k, \theta) \ll \Omega_{j}$. In this case, the mutual friction does not significantly affect the energy balance, and we expect the energy spectrum $\tilde{F}_{j j}(k, \theta)$ to be close to the Kolmogorov 1941 prediction $E_{\mathrm{K} 41}(k) \propto k^{-5 / 3}$ for both components. For large $k$ and with $\cos \theta \sim 1$, the velocity components are almost decoupled $D(k, \theta) \ll 1$, and the mutual-friction energy dissipation is maximal: $\mathcal{D}_{j}^{\mathrm{mf}}(k, \theta) \approx \Omega_{j} \tilde{F}_{j j}(k, \theta)$. This situation is similar to that in ${ }^{3} \mathrm{He}$ with the normal-fluid component at rest [13]. In such a case, we can expect that the energy dissipation by mutual friction strongly suppresses the energy spectra, much below $E_{\mathrm{K} 41}(k)$.

Combining all these considerations, we expect the energy spectra $\tilde{F}_{j j}(k, \cos \theta)$ to become more anisotropic with increasing $k$, with most of the energy concentrated in the range of small $\cos \theta$, i.e., in the orthogonal plane.

Numerical results.-Direct numerical simulations of the coupled Eqs. (1a) and (1b) were carried out using a fully dealiased pseudospectral code with a resolution of $256^{3}$ collocation points in a triply periodic domain of size $L=2 \pi$. To reach a steady-state flow, velocity fields of the normalfluid and superfluid components are stirred by two independent random Gaussian forces $\varphi_{\mathrm{s}}$ and $\varphi_{\mathrm{n}}$ with the force amplitudes $|\varphi|=0.5$ for both components, localized in the band $k_{\varphi} \in[0.5,1.5]$. The time integration is performed using a second-order Adams-Bashforth scheme with the viscous term exactly integrated.

We have decided to focus on the temperature $T=1.85 \mathrm{~K}$, at which the densities and viscosities of the normal-fluid and superfluid components are close: $\rho_{\mathrm{s}} / \rho_{\mathrm{n}}=$ 1.75 and $\nu_{\mathrm{s}} / \nu_{\mathrm{n}}=1.07$. The mutual friction parameter for this temperature is $\alpha=0.18$. The simulations were carried out with both the normal-fluid and superfluid viscosity $\nu_{\mathrm{n}}=\nu_{\mathrm{s}}=0.003$. Other parameters of the simulations were chosen based on the relevant dimensionless relations: the Reynolds numbers and the normal-fluid turbulent intensity $w: \operatorname{Re}_{j}=\left(u_{\mathrm{T}}^{j}\right)\left(/ \nu_{j} k_{0}\right), w=U_{\mathrm{ns}} / u_{\mathrm{T}}^{\mathrm{n}}$. Here $u_{\mathrm{T}}^{j}=\sqrt{\left\langle u_{j}{ }^{2}\right\rangle}$ is the root mean square (rms) of the turbulent velocity fluctuations, and $k_{0}=1$ is the outer scale of turbulence. To emphasize the importance of the counterflow, we compare the results with the simulations for the so-called coflow with the rest of the parameters being the same. In the coflow, the two components of the mechanically driven ${ }^{4} \mathrm{He}$, being coupled by the mutual friction force, move in the same direction with the same mean velocities, $U_{\mathrm{ns}}=0$. The statistics in the coflow configuration is known to be similar to that of classical isotropic turbulence [14,27-29]. In our simulations, the values of the Reynolds numbers in the counterflow are $\operatorname{Re}_{\mathrm{n}}=1051$ and $\operatorname{Re}_{\mathrm{s}}=1056$, while in the coflow, $\operatorname{Re}_{\mathrm{n}}=1179$ and $\operatorname{Re}_{\mathrm{s}}=1181$. The rms velocities of both components in both flows are $u_{\mathrm{T}}^{\mathrm{s}}=u_{\mathrm{T}}^{\mathrm{n}}=3.5$. The dimensionless values of the mutual friction frequency $\Omega_{\mathrm{s}}=$ 20 and the counterflow velocity $U_{\mathrm{ns}}=15.4$ correspond to the case with both components strongly turbulent and strongly coupled. The results on the temperature and $\Omega_{\mathrm{s}}$ dependence of the energy spectra will be reported elsewhere. The flow conditions were controlled by the simulations of the uncoupled equations without counterflow $\left(\boldsymbol{U}_{j}=\boldsymbol{\Omega}_{j}=0\right)$, which represent here the classical hydrodynamic isotropic turbulence (CHT).

The energy spectra are influenced by the viscous dissipation, by the dissipation due to mutual friction, and by the counterflow-induced decoupling. To clarify the role of each of these factors, we first ignore the expected anisotropy and compare in Fig. 2(a) the normal-fluid and superfluid energy spectra $E_{\mathrm{nn}}(k)$ and $E_{\mathrm{ss}}(k)$ and the cross-correlation $E_{\mathrm{ns}}(k)$, integrated over a spherical surface of radius $k$, i.e., over all directions of vector $\boldsymbol{k}$ : 

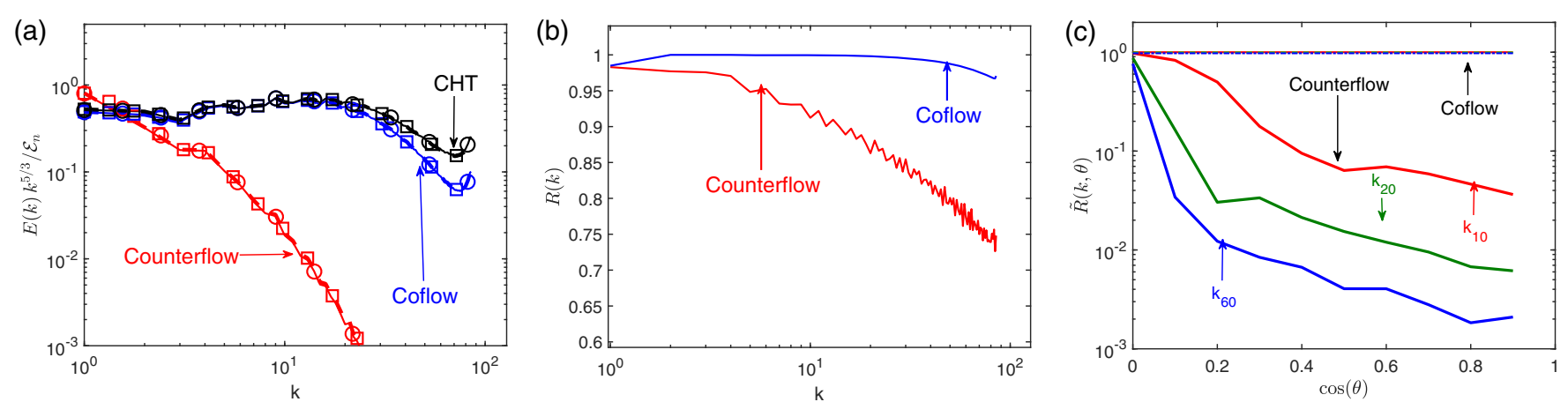

FIG. 2. (a) The spherical energy spectra $E_{j j}(k)$ of the normal fluid (circles) and the superfluid (squares). (b) The cross-correlation function $R(k)$. (c) The angular dependence of the cross-correlation function $\tilde{R}(k, \theta)$ for the coflow and the counterflow. In panel (c), all data for the coflow coincide. For the counterflow, red lines correspond to $\tilde{R}(k, \theta)$ averaged over the wave number range $10 \leq k<20$, green lines to the averaging over $20 \leq k<60$, and blue lines to the averaging over $60 \leq k \leq 80$ (labeled as $k_{10}, k_{20}$, and $k_{60}$, respectively). Note the log-linear scale.

$$
E_{i j}(k)=\int \mathcal{F}_{i j}(\boldsymbol{k}) \frac{d \phi d \cos \theta}{(2 \pi)^{3}}
$$

The corresponding normalized cross-correlation functions

$$
R(k)=2 E_{\mathrm{ns}}(k) /\left[E_{\mathrm{nn}}(k)+E_{\mathrm{ss}}(k)\right]
$$

are shown in Fig. 2(b). The effect of viscous dissipation is clearly seen in the spectra of the uncoupled components, corresponding to CHT (marked "CHT," black lines). The spectra almost coincide, since at $T=1.85 \mathrm{~K}$ the viscosities are close. In the coflow, the strongly coupled components are well correlated at all scales and move almost as one fluid. Note the additional dissipation due to mutual friction, leading to further suppression of the spectra compared to the uncoupled case. The presence of the counterflow velocity leads to a sweeping [12] of the two components' eddies in opposite directions by the corresponding mean velocities. The result is the decorrelation of the components' turbulence velocities, especially at small scales, for which the overlapping time is very short, see Fig. 2(b). The dissipation by mutual friction is very strong in this case, with both $\Omega$ and the velocity difference being large, leading to very strongly suppressed spectra, with $E_{\mathrm{nn}}(k) \approx E_{\mathrm{ss}}(k)$. This behavior was predicted by the theory [15], based on the assumption of spectral isotropy. However, the spherically integrated spectra and cross-correlations cannot reveal any properties connected to the anisotropic action of the mutual friction force. To account for the spectral anisotropy, we plot in Fig. 2(c) the normalized 2D cross-correlations

$$
\tilde{R}(k, \theta)=2 \tilde{F}_{\mathrm{ns}}(k, \theta) /\left[\tilde{F}_{\mathrm{nn}}(k, \theta)+\tilde{F}_{\mathrm{ss}}(k, \theta)\right] .
$$

Given the discrete nature of the $\boldsymbol{k}$ space in DNS, we average them over three bands of wave numbers. Leaving aside $k \approx k_{0}$, influenced by the forcing, we average $\tilde{R}(k, \theta)$ over the $k$ ranges $10 \leq k<20,20 \leq k<60$, and $60 \leq k \leq 80$.
The first observation here is that the cross-correlation for the coflow are isotropic at all scales (see the thin horizontal lines, marked "coflow"). On the other hand, in the counterflow, the cross-correlations are largest for $\cos \theta \approx$ 0 and fall off very fast with decreasing angle, slower for small $k$ (red lines, labeled $k_{10}$ ) and faster as $k$ becomes larger (green and blue lines, $k_{20}$ and $k_{60}$, respectively). Such a strong decorrelation of the components velocities leads to an enhanced dissipation by mutual friction in the counterflow direction, such that most of the energy is contained in the narrow range $\cos \theta \lesssim 0.1$, near the plane orthogonal to $\boldsymbol{U}_{\mathrm{ns}}$. Indeed, the superfluid energy spectrum $F_{\text {ss }}\left(k_{\|}, k_{\perp}\right)$, shown in Fig. 3(a), is strongly suppressed in the $k_{\|}$direction, while it decays slowly in the orthogonal plane. A similar phenomenon of the creation of quasi-2D turbulence is observed in a strongly stratified atmosphere [30-32] and in rotating turbulence [33-35], where there exists a preferred direction defined by gravity or by a rotation axis. The difference between these examples and the present counterflow lies in the nature of the velocity field. The leading velocity components in the classical flows are in a plane orthogonal to the preferred direction. Moreover, at small scales the isotropy is restored [31,32]. On the contrary, in ${ }^{4} \mathrm{He}$ counterflow, the dominant velocity component is oriented along the
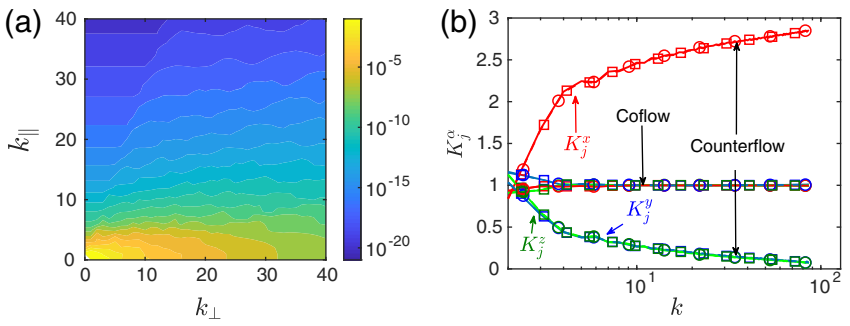

FIG. 3. (a) The superfluid component energy spectrum $F_{\text {ss }}\left(k_{\|}, k_{\perp}\right)$ in the counterflow. (b) The tensor decomposition of the normalized spherical energy spectra $K_{j}^{\alpha}(k)$ for the normal fluid (circles) and the superfluid (squares). 
counterflow direction, with the anisotropy becoming stronger with decreasing scales, see Fig. 3(b). Here we plot the tensor components of the spherical spectra as the ratios

$$
K_{j}^{\alpha}(k) \equiv 3 E_{j j}^{\alpha \alpha}(k) / E_{j j}(k) .
$$

The factor 3 was introduced to ensure that for isotropic turbulence, $K_{j}^{\alpha}(k)=1$. Expectedly, the coflow (the almost horizontal lines) is isotropic at all scales, except for the smallest wave numbers. On the other hand, for the counterflow turbulence, the contribution of the $K_{j}^{x}(k)$ component (shown by red lines) is dominant and monotonically increases with $k$ from the isotropic level $K_{j}^{x}\left(k_{0}\right) \approx 1$ to the maximal possible level, $K_{j}^{x}(k) \approx 3$. Therefore, the smallscale counterflow turbulence consists mainly of $v_{j}^{x}(k)$ velocity fluctuations. The contribution of $v_{j}^{y}$ and $v_{j}^{z}$ fluctuations for $k \gtrsim 10$ is negligible. Summarizing Fig. 3, the leading contribution to the spectra of small-scale counterflow turbulence comes from the turbulent velocity fluctuations with only one streamwise projection that depends on the two cross-stream coordinates $\{y, z\}: u^{x}(y, z)$. Such turbulence can be visualized as narrow jets or thin sheets with velocity, oriented along the counterflow and randomly distributed in the orthogonal plane. Indeed, the velocity components $u_{\mathrm{s}}^{y}$ (shown in Fig. 1) and $u_{\mathrm{s}}^{z}$ have only large-scale structures, while $u_{\mathrm{s}}^{x}$ has elongated structures at various scales.

Summary.-The energy spectra of the superfluid ${ }^{4} \mathrm{He}$ counterflow turbulence become more anisotropic upon going from large scales toward scales about the intervortex distance. This strong anisotropy distinguishes it from the classical turbulent flows that become more isotropic as the scale decreases. Most of the turbulent energy becomes concentrated in the plane, orthogonal to the counterflow direction. Furthermore, contrary to classical quasi-2D turbulent flows in rotation or in stratified configurations, where dominant velocity components lie in the same plane, the only surviving velocity component at small scales is preferentially oriented along the counterflow direction. The selective suppression of the orthogonal velocity fluctuations has its origin in the strong anisotropy of the energy dissipation by mutual friction, resulting from the angular dependence of the components' cross-correlation. The anisotropy of the energy spectra can be measured once information from different spatial positions is available. One can think of using particle imaging velocimetry [36] to detect the velocity field in the streamwise-wall-normal plane, a pair of very small second sound sensors, placed at some separation in the wall-normal direction [37], or a particle's visualization at separate streamwise locations $[26,38]$. Such methods allow us to measure the velocity fluctuations in different directions and to reconstruct the energy spectra along and across the counterflow direction.

L. B. acknowledges funding from the European Union's Seventh Framework Programme (FP7/2007-2013) Grant
No. 339032. G. S. thanks the AtMath Collaboration at the University of Helsinki. D. K. acknowledges funding from the Simons Foundation under Grant No. 454955 (Francesco Zamponi).

[1] L. Biferale and I. Procaccia, Phys. Rep. 414, 43 (2005).

[2] U. Frisch, Turbulence: The Legacy of A.N. Kolomogorov (Cambridge University Press, Cambridge, England, 1995).

[3] R. J. Donnelly, Phys. Today 62, No. 10, 34 (2009).

[4] R. J. Donnelly, Quantized Vortices in Hellium II (Cambridge University Press, Cambridge, England, 1991).

[5] W. F. Vinen and J. J. Niemela, J. Low Temp. Phys. 128, 167 (2002).

[6] H. E. Hall and W. F. Vinen, Proc. R. Soc. A 238, 204 (1956).

[7] W. F. Vinen, Proc. R. Soc. 240, 114 (1957); 240, 128 (1957); 242, 493 (1957); 243, 400 (1958).

[8] R. N. Hills and P. H. Roberts, Arch. Ration. Mech. Anal. 66, 43 (1977).

[9] Quantized Vortex Dynamics and Superfluid Turbulence, edited by C. F. Barenghi, R. J. Donnelly, and W. F. Vinen, Lecture Notes in Physics Vol. 571 (Springer-Verlag, Berlin, 2001).

[10] R. P. Feynman, Prog. Low Temp. Phys. 1, 17 (1955).

[11] L. Boue, V. S. L'vov, Y. Nagar, S. V. Nazarenko, A. Pomyalov, and I. Procaccia, Phys. Rev. B 91, 144501 (2015).

[12] D. Khomenko, V. S. L'vov, A. Pomyalov, and I. Procaccia, Phys. Rev. B 93, 014516 (2016).

[13] L. Biferale, D. Khomenko, V. S. L'vov, A. Pomyalov, I. Procaccia, and G. Sahoo, Phys. Rev. B 95, 184510 (2017).

[14] L. Biferale, D. Khomenko, V. S. L'vov, A. Pomyalov, I. Procaccia, and G. Sahoo, Phys. Rev. Fluids 3, 024605 (2018).

[15] V. S. L'vov and A. Pomyalov, Phys. Rev. B 97, 214513 (2018).

[16] V. S. L'vov, S. V. Nazarenko, and G. E. Volovik, JETP Lett. 80, 535 (2004).

[17] R. J. Donnelly and C. F. Barenghi, J. Phys. Chem. Ref. Data 27, 1217 (1998).

[18] D. Khomenko, V. S. L'vov, A. Pomyalov, and I. Procaccia, Phys. Rev. B 93, 134504 (2016).

[19] S. B. Pope, Turbulent Flows (Cambridge University Press, Cambridge, England, 2000).

[20] S. K. Nemirovskii and V. V. Lebedev, Sov. Phys. JETP 57, 1009 (1983).

[21] T. Lipniacki, Phys. Rev. B 64, 214516 (2001).

[22] D. Jou, M.S. Mongioví, and M. Sciacca, Physica (Amsterdam) 240D, 249 (2011).

[23] D. Khomenko, V. S. L'vov, A. Pomyalov, and I. Procaccia, Phys. Rev. B 97, 014508 (2018).

[24] E. Varga, J. Gao, W. Guo, and L. Skrbek, Phys. Rev. Fluids 3, 094601 (2018).

[25] J. Gao, E. Varga, W. Guo, and W. F. Vinen, Phys. Rev. B 96, 094511 (2017).

[26] S. Bao, W. Guo, V. S. L'vov, and A. Pomyalov, Phys. Rev. B 98, 174509 (2018).

[27] L. Skrbek and K. R. Sreenivasan, in Ten Chapters in Turbulence, edited by P. A. Davidson, Y. Kaneda, and K. R. Sreenivasan (Cambridge University Press, Cambridge, England, 2013), pp. 405-437. 
[28] C. F. Barenghi, V. S. L'vov, and P.-E. Roche, Proc. Natl. Acad. Sci. U.S.A. 111, 4683 (2014).

[29] E. Rusaouen, B. Chabaud, J. Salort, and P.-E. Roche, Phys. Fluids 29, 105108 (2017).

[30] E. J. Hopfinger, J. Geophys. Res. 92, 5287 (1987).

[31] A. Kumar, M. K. Verma, and J. Sukhatme, J. Turbul. 18, 219 (2017).

[32] A. Alexakis and L. Biferale, Phys. Rep. 767-769, 1 (2018).

[33] L. Biferale, F. Bonaccorso, I. M. Mazzitelli, M. A. T. van Hinsberg, A. S. Lanotte, S. Musacchio, P. Perlekar, and F. Toschi, Phys. Rev. X 6, 041036 (2016).
[34] B. Gallet, A. Campagne, P.-P. Cortet, and F. Moisy, Phys. Fluids 26, 035108 (2014).

[35] B. Gallet, J. Fluid Mech. 783, 412 (2015).

[36] D. W. Carter and F. Coletti, J. Fluid Mech. 827, 250 (2017).

[37] P.-E. Roche, P. Diribarne, T. Didelot, O. Français, L. Rousseau, and H. Willaime, Europhys. Lett. 77, 66002 (2007).

[38] F. Wang, I. Toselli, J. Li, and O. Korotkova, Opt. Lett. 42, 1129 (2017). 